\section{IDDF2020-ABS-0016 CLINICOPATHOLOGIC CHARACTERISTICS AND TREATMENT OUTCOMES OF RECTAL GASTROINTESTINAL STROMAL TUMORS: EXPERIENCE FROM A TERTIARY CENTER}

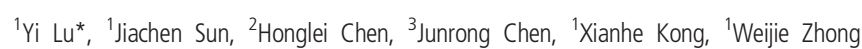
${ }^{1}$ Department of Gastrointestinal Endoscopy, the Sixth Affiliated Hospital, Sun Yat-sen University, China; ${ }^{2}$ Department of Gastrointestinal Endoscopy, the Eighth Affiliated Hospital, Sun Yat-sen University, China; ${ }^{3}$ Department of Gastroenterology, the Sixth Affiliated Hospital, Sun Yat-sen University, China

\subsection{6/gutjnl-2020-IDDF.43}

Background To investigate the clinicopathologic characteristics, surgical and imatinib management and long-term follow-up outcomes of the rectal gastrointestinal stromal tumors (GISTs). Methods Consecutive patients with rectal GISTs admitted in our center (from January 2013 to June 2018) were chosen. Their history information was viewed, and the follow-up results were obtained by phone or medical records.

Results Forty-nine patients (32males and 17 females) were identified, with a median age of 59 years, and 36 patients received surgery. Most (46 patients, 93.9\%) of the tumor were located within $6 \mathrm{~cm}$ from the anal verge, 18 patients $(36.7 \%)$ had very low or low risk, and 31patients $(63.3 \%)$ had intermediate or high risk. Four kinds of surgery approach were applied in our center: trans-abdominal ( 8 patients, 22.2\%), trans-anal/trans-perineal (15 patients, $41.7 \%)$, transsacral (12 patients, $33.3 \%$ ) and abdominal perineal (1 patient, $2.8 \%)$. The complication is low, and the mortality related to surgery is $0 \%$. After a median follow-up of 705 days (ranged from 48 days to 1677 days), 3 patients (8.33\%) were found to have a recurrence.

Conclusions Trans-anal/trans-perineal and trans-sacral surgery were more commonly used in our study, and for now, the recurrence rate had no difference, but longer time for followup time is needed.

\section{IDDF2020-ABS-0020 MONITORING ADHERENCE TO GLUTEN- FREE DIET USING MEAN PLATELET VOLUME IN CHILDREN WITH CELIAC DISEASE}

${ }^{1}$ Deepak Kumar*, ${ }^{2}$ Garima Gupta. 'Assistant Professor, Maulana Azad Medical College, India; ${ }^{2}$ Government, India

\subsection{6/gutjnl-2020-IDDF.44}

Background Celiac disease was thought to be uncommon, but now cases are prevalent worldwide. It is an autoimmune disease with the genetic susceptibility of the patient to glutencontaining food. The only treatment available is a gluten free diet. Compliance to the gluten-free diet and its monitoring becomes important and is difficult to follow in the pediatric patients. There are various invasive and non-invasive investigations available to monitor compliance. However, their availability and cost always remain an issue at low-resource health set-ups. MPV (Mean Platelet Volume) is a non-invasive, economic and easily available marker that can be used safely.

Methods Mean Platelets volume was measured on newly diagnosed celiac disease children between 3 to 12 years at baseline and after 3 months of starting Gluten-free diet. Total no. of patients $n=35, M: F$ ratio- 1.1 . Recurrent diarrhea (85\%), pain abdomen (50\%) and distension (45\%) were the common abdominal symptoms. Anemia, Short stature and malnutrition were present in more than $90 \%$ of patients. The screening was done with TTG, and cases were confirmed with Duodenal biopsy. Marsh 3A was present in 5\%, 3B in 52\% and $3 \mathrm{C}$ in 43\%. Mean Baseline MPV was $9.55 \mathrm{fL}$.

Results Patients were started on a gluten-free diet, they were followed monthly for symptoms or any new event, at the end of 3 months of follow up MPV was done, we observed that MPV was decreased from mean baseline level of $9.55 \mathrm{fL}$ to 8.42 fL. (P 0.001).

Conclusions Reversibility of tissue changes after introduction to gluten-free diet remains the gold standard to see the improvement. However, it is an invasive procedure and an impractical method to follow up, especially in children. Other markers are not easily available at all health centers, specifically at developing and underdeveloped parts of the world. MPV can be used as a biomarker to see diet adherence and improvement in patients suffering from Celiac Disease. It has a disadvantage that high values can be found if there is associated co-infection or Iron Deficiency Anemia, which should be corrected before making any interpretation.

\section{IDDF2020-ABS-0021 A META-ANALYSIS OF EFFICACY OF TOPICAL STEROIDS IN EOSINOPHILIC ESOPHAGITIS: FROM THE PERSPECTIVE OF HISTOLOGIC, CLINICAL, AND ENDOSCOPIC OUTCOME}

${ }^{1}$ Lixiao Hao*, ${ }^{2} Y_{i}$ Lu, ${ }^{1}$ Tao Li. ${ }^{1}$ Department of Digestive Endoscopy, Shanghai Shuguang Hospital, Shanghai University of Chinese Medicine, China; ${ }^{2}$ Department of Gastrointestinal Endoscopy, the Sixth Affiliated Hospital, Sun Yat-sen University, China

\subsection{6/gutjin-2020-IDDF.45}

Background Swallowed topical steroids are a mainstay of eosinophilic esophagitis (EoE) drug therapy, studies showed a good histologic response, while with a great discrepancy in clinical and endoscopic improvement. We conducted this meta-analysis to investigate the efficacy of topical steroids in EoE in histological response, clinical and endoscopic improvement.

Methods Several databases were searched from inception to 1st August 2019 for randomized controlled trials (RCTs) comparing topical steroids with placebo for EoE in short-term. The outcomes of interest mainly included basic characteristics of the studies, histologic, clinical, endoscopic response rate and adverse events. Inconsistency was quantified using $I^{2}$ statistics.

Results Nine studies were eventually selected for the final analysis. The meta-analysis showed that topical steroids were effective in inducing histologic response compared with placebo both for complete response (OR 35.82, 95\% CI 14.98-85.64, $\left.\mathrm{P}<0.0001 ; I^{2}=0, \mathrm{P}=0.72\right)$ and partial response (OR 28.44, 95\%CI 8.56-94.47, $\mathrm{P}<0.0001 ; I^{2}$ $=70 \%, \mathrm{P}=0.0009)$. Also, topical steroids were useful in gaining clinical response (OR 2.53, 95\%CI 1.14-5.60, $\left.\mathrm{P}=0.02 ; I^{2}=60 \%, \mathrm{P}=0.02\right)$ and endoscopic response $((\mathrm{OR}$ 3.51 , 95\%CI $\left.\left.1.47-8.36, \mathrm{P}=0.005 ; I^{2}=0, \mathrm{P}=0.57\right)\right)$. Generally, topical steroids are well tolerated. The most common adverse event is infections and infestations (59 cases), and oropharyngeal or esophageal candidiasis is the most common infection (24 cases).

Conclusions Topical steroids were effective in inducing a histological, clinical and endoscopic response in short-term, and 